\title{
Dynamic Analysis of Eddy Current Damping Mechanism Employing 3-D Finite Element Method
}

$\begin{array}{ll}\text { Katsuhiro Hirata }^{*} & \text { Senior Member } \\ \text { Yuya Hasegawa** }^{* *} & \text { Non-member } \\ \text { Tomohiro Ota }^{* *} & \text { Member } \\ \text { Tadashi Yamaguchi }^{* * *} & \text { Member } \\ \text { Yoshihiro Kawase }^{* * *} & \text { Senior Member } \\ \text { Takafumi Eguchi }^{* * *} & \text { Non-member } \\ \text { Hiroyuki Kodama }^{* * *} & \text { Non-member }\end{array}$

This paper describes a dynamic response analysis of a laser marker which has an eddy current damping mechanism. In this calculation the equation of magnetic field is coupled with the equation of motion employing the 3-D finite element method (FEM), and time variations of the pendulum are computed to estimate the damping time. The usefulness of the computation is clarified through the comparison with the measured results. Moreover, the effectiveness of proposed damping structures is also confirmed by the comparison with the conventional one.

Keywords: dynamic response analysis, damping mechanism, 3-D finite element method, eddy current

\section{Introduction}

Recently, laser markers ${ }^{(1)}$ have been used at the construction spot because they are very convenient to set both horizontal and vertical base lines. Most of middle range laser markers adopt eddy current force to control the oscillation of the pendulum because of its structural simplicity. One of the most important characteristics of laser markers is the damping time after they are set on the ground, and various kinds of damping structure have been developed.

The computer simulation is very important in optimizing the geometry of the damping structure, but the 3-D dynamic analysis is thought to be difficult to directly apply to a complicated large model in actual design. Authors have been studying useful techniques (2)(3) ${ }^{(2)}$ dynamic analysis to save the CPU time.

In this paper, the 3-D eddy current damping analysis ${ }^{(4)}$ coupled with the equation of motion is applied to design the damping mechanism of a laser marker. The validity of this computation is confirmed by the comparison with the measured results.

Furthermore, we proposed a few effective damping structures so that the oscillation of the pendulum can be quickly decreased. The effectiveness of the proposed structures are clarified by the comparison with the conventional one.

\footnotetext{
* Department of Adaptive Machine Systems Graduate School of Engineering, Osaka University

2-1, Suita, Osaka 565-0871

** Analytical Evaluation \& Reliability Technology Center, Matsushita Electric Works, Ltd.

1048, Kadoma, Osaka 571-8686

*** Department of Information Science, Gifu University

1-1, Yanagido, Gifu 501-1193
}

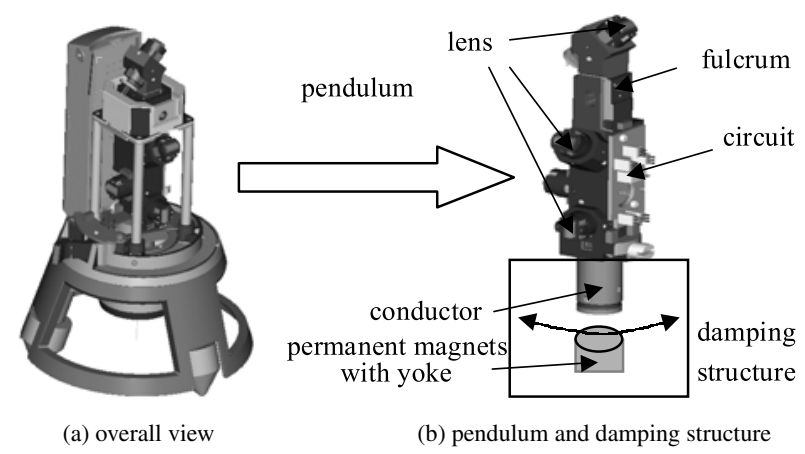

Fig. 1. Basic construction of this laser maker

\section{Damping Structure of Laser Marker}

Fig. 1 shows the basic construction of the laser marker. This system uses the eddy current damping mechanism to control the oscillation of the pendulum. The dumping structure is mostly composed of the conductor (cupper) at the bottom of the pendulum and the permanent magnets with yoke. The pendulum swings at the center of the fulcrum and the eddy current force acts on a cupper conductor. It is desired to control the oscillation of pendulum within five seconds.

\section{Analysis Method}

3.1 Magnetic Field Analysis The fundamental equations of the magnetic field can be expressed using the magnetic vector potential $\boldsymbol{A}$ and the electric scalar potential $\phi$ as follows.

$$
\operatorname{rot}(v \operatorname{rot} \boldsymbol{A})=\boldsymbol{J}_{e}+v_{0} \operatorname{rot} \boldsymbol{M}
$$




$$
\begin{aligned}
& \boldsymbol{J}_{e}=-\sigma\left(\frac{\partial \boldsymbol{A}}{\partial t}+\operatorname{grad} \phi\right) \\
& \operatorname{div} \boldsymbol{J}_{e}=0 \ldots \ldots \ldots \ldots
\end{aligned}
$$

where $v$ is the reluctivity, $\boldsymbol{J}_{e}$ is the eddy current density, $v_{0}$ is the reluctivity of the vacuum, $\boldsymbol{M}$ is the magnetization of the permanent magnet, and $\sigma$ is the conductivity.

3.2 Calculation of Eddy Current Force The eddy current force actiong on the conductor is calculated by the following equation of Lorentz force.

$$
\boldsymbol{F}=\int_{V} \boldsymbol{f} d v, \boldsymbol{f}=\boldsymbol{J}_{e} \times \boldsymbol{B}
$$

where $f$ is the Lorentz force per the unit volume, $V$ is the region of the conductor, and $\boldsymbol{B}$ is the flux density.

3.3 Coupled Analysis with Motion Equation The equation of motion is coupled with that of the magnetic field in this analysis. The movement of a conductor is supposed to be linear motion ( $x$-direction), because the length of pendulum from the center of the fulcrum is long enough as compared with the displacement of x-direction. In order to confirm the validity of this approximation, Lorentz forces at the gap length of $0.85,1.0$, and $1.15 \mathrm{~mm}$ are compared when the pendulum is forced to move with sinusoidal oscillation $( \pm 5 \mathrm{~mm}, 1.8 \mathrm{~Hz})$. As the results, it is found that the change of average Lorentz force is about $5 \%$, and then the damping time is not influenced (less than 1\%). Then, the eqation of motion is derived as follows.

$$
M \frac{d^{2} x}{d t^{2}}=\mp F_{x} \pm F_{k} \mp F_{s}
$$

where $M$ is the mass of the pendulum, $F_{x}$ is the component of $x$-direction of the Lorentz force, $F_{k}$ is the restoring force, and $F_{s}$ is the viscous drag.

$F_{k}$ in (5) can be approximated as follows.

$$
F_{k}=M g \sin \theta \fallingdotseq M g \frac{x}{l}
$$

where $g$ is the acceleration of gravity, and $l$ is the length of pendulum from the fulcrum.

$F_{s}$ in (5) is given by the coefficient of viscosity $C$ as follows.

$$
F_{s}=C \frac{d x}{d t}
$$

\section{Results and Discussion}

4.1 Comparison between Proposed and Conventional Models Fig. 2 shows aspect of parts of the proposed damping structure. The stator of damping structure has dual permanent magnets magnetized by an opposite direction and attached to the common yoke so that it can produce highly gradient of magnetic field with respect to the conductor displacement $(d \phi / d x)$. Fig. 3 and Table 1 show the analyzed model and the conditions, respectively.

Fig. 4 shows the calculated and measured time variations of the displacement in using the proposed damping structure model. It is found that both results are in good agreement. Fig. 5 shows the distributions of the flux density vectors, the eddy current density vectors and the Lorentz force vectors when the velocity of moving conductor is the maximum. It

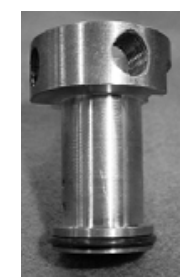

(a) conductor (copper)

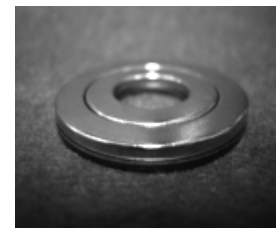

(b) stator (permanent magnets with yoke)
Fig. 2. Aspect of parts of the proposed damping structure

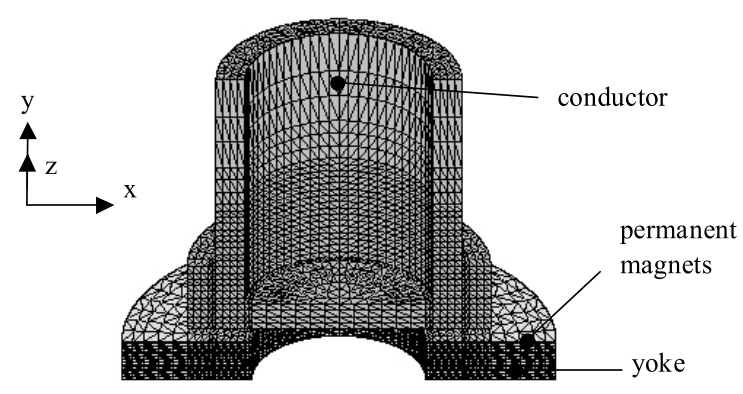

(a) $d=0.0 \mathrm{~mm}$

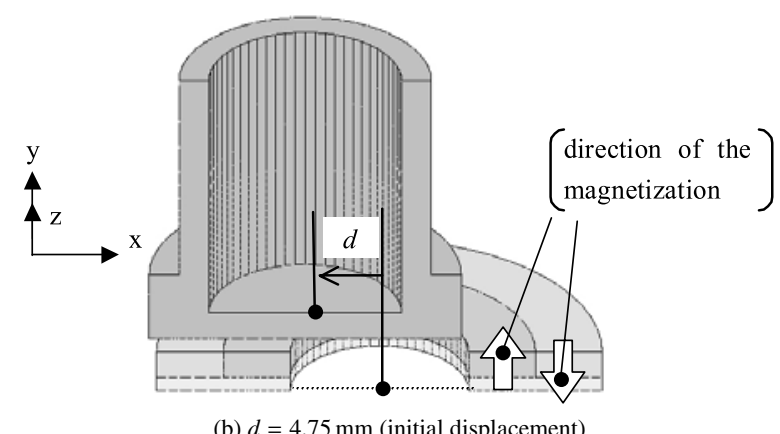

Fig. 3. Analyzed model (proposed model)

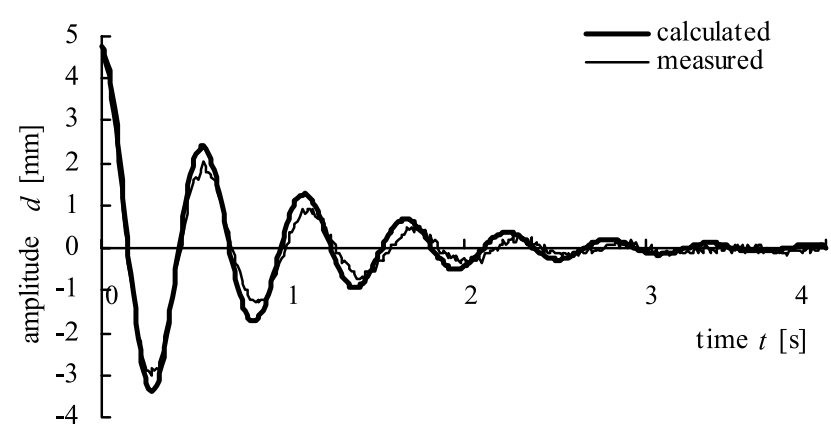

Fig. 4. Calculated and measured time variations of the proposed model

Table 1. Analyzed conditions

\begin{tabular}{c|c}
\hline \hline Magnetization of magnet $[\mathrm{T}]$ & 1.36 \\
\hline Conductivity of conductor [S/m] & $5.8^{\times} 10^{7}$ \\
\hline Gap length between conductor and magnets [mm] & 1.0 \\
\hline Mass of pendulum [g] & 190 \\
\hline Length of pendulum [mm] & 78 \\
\hline Initial displacement of conductor [mm] & 4.75 \\
\hline Coefficient of viscosity $[\mathrm{N} \cdot \mathrm{s} / \mathrm{m}]$ & 0.15 \\
\hline \hline
\end{tabular}




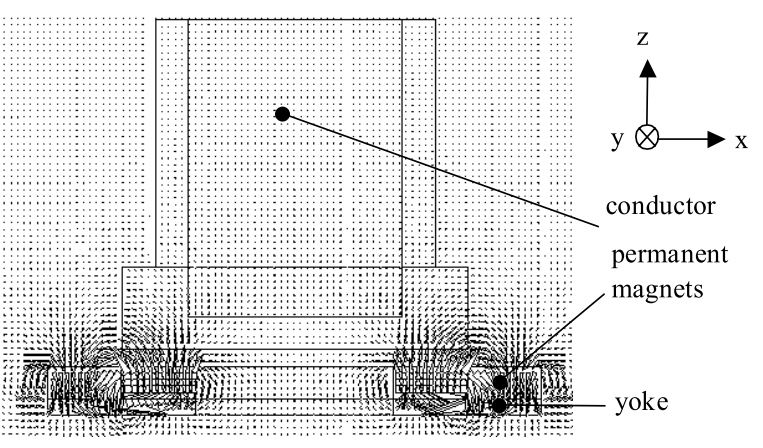

(a) flux density vectors

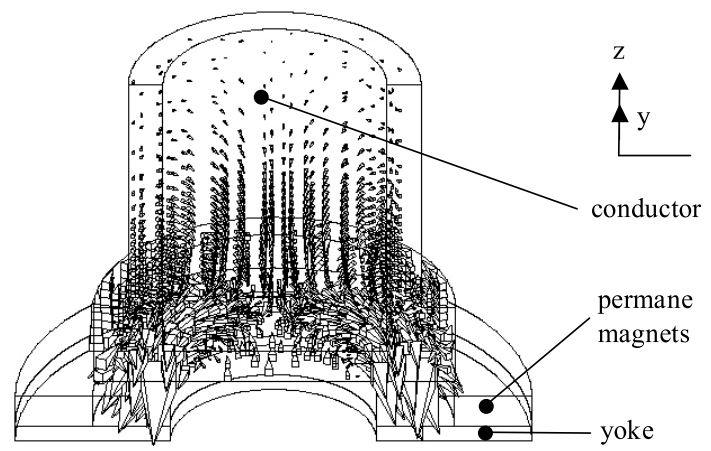

(b) eddy current density vectors

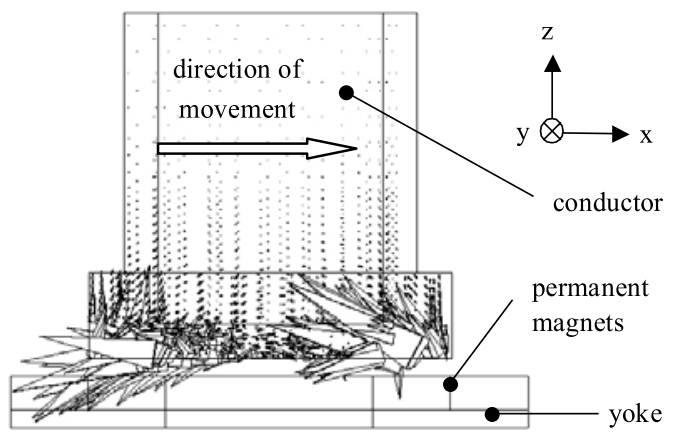

(c) Lorentz force density vectors

Fig. 5. Distributions of flux density vectors, eddy current density vectors and Lorentz force density vectors of the proposed model

is found that the eddy current greatly flows in the conductor above the boundary of dual magnets where the gradient of magnetic field with respect to the position is the maximum, and strong Lorentz force acts on a conductor as an eddy current-brake in the opposite direction of the conductor movement.

Next, the effectiveness of the proposed model is confirmed through the comparison with a conventional damping structure model. Fig. 6 shows the basic construction of the conventional model. It has the same outer diameters of the magnet and conductor as the proposed one. The masses of both models are also the same. The structural difference is that the conventional model has only a single magnet in the stator and has no brim at the bottom of the cupper conductor. The influences of geometry parameters on the damping time were investigated. As the results, the influences of the center hole of the conductor and the stator side yoke were found to be negligible from the analyzed results. In both models the diameter of brim and magnet layout are optimized to obtain the best damping characteristics. Dual magnet model has

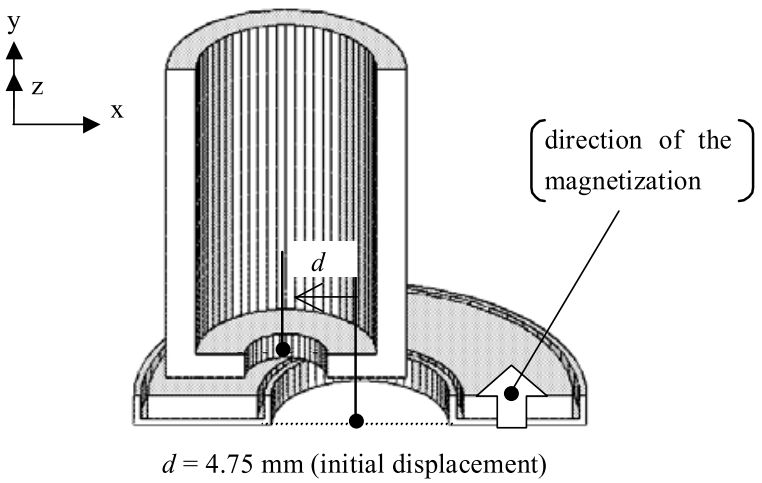

Fig. 6. Basic construction of the conventional model

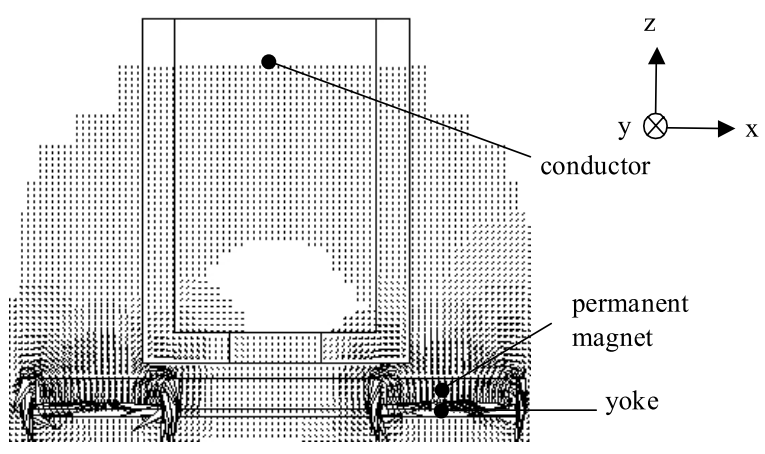

(a) flux density vectors

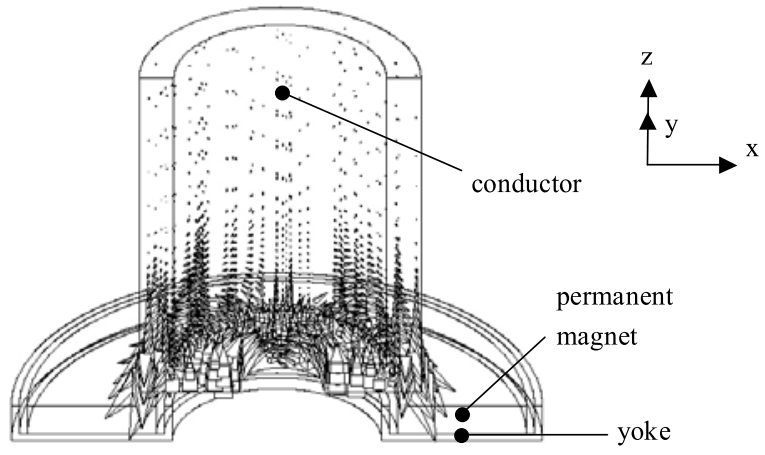

(b) eddy current density vectors

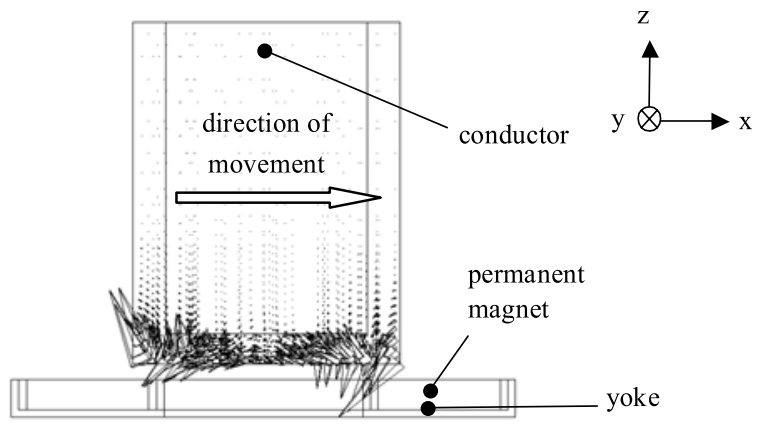

(c) Lorentz force density vectors

Fig. 7. Distributions of flux density vectors, eddy current density vectors and Lorentz force density vectors of the conventional model

the same outer diameter of brim as that of the inner magnet (boundary of two magnets) because the Lorentz force is efficiently generated through this boundary where the gradient of magnetic field against the displacement becomes peak.

Dynamic response of the conventional model is computed 
in completely the same analyzed conditions as the proposed model mentioned above. Fig. 7 shows the distributions of the flux density vectors, the eddy current density vectors and the Lorentz force vectors of conventional damping model. As shown in this figure, flux density vectors in the conductor are very small because the magnetic circuit is not efficient as compared with that of the proposed one, and the very small and localized vectors of eddy currents and Lorentz force are seen in Fig. 7(b), (c).

As shown in Fig. 8, it is found that the proposed damping model can amazingly control the oscillation of the pendulum compared with the conventional one. The damping time of the proposed model is about 3 seconds though the conventional model needs over 50 seconds.

Fig. 9 shows the computed steady-state waveforms of Lorentz force of both models when the conductor is forced to move with sinusoidal oscillation $( \pm 5 \mathrm{~mm}, 1.8 \mathrm{~Hz})$. It is found that the proposed model gives about 2.7 times as big average damping force as the conventional one.

4.2 Another Proposed Model Fig. 10 shows another proposed model (sandwiched model), in which diskshaped moving conductor is inserted between two stators to control the magnetic flux vectors efficiently. The analyzed conditions are the same as other analyzed models mentioned above (see Table 1). Fig. 11 shows the distributions of the flux density vectors, the eddy current density vectors and the Lorentz force vectors when the velocity of the conductor is the maximum. As compared with Fig. 5, it is found that the

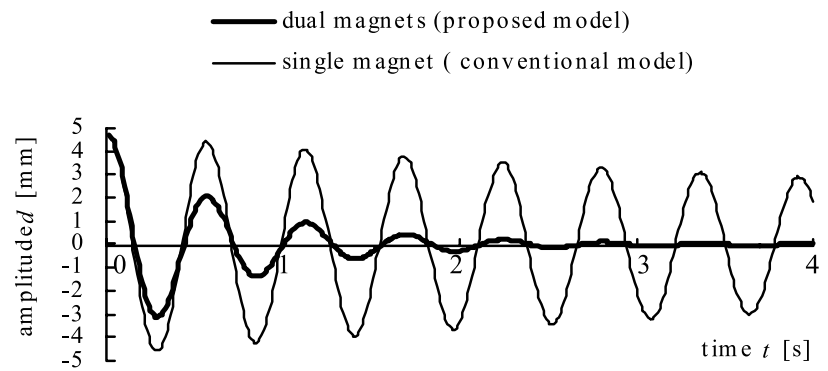

Fig. 8. Comparison of dynamic response between the proposed and conventional models

$$
\begin{aligned}
& -\infty \text { Lorent } z \text { force (conventionall) } \\
& -\Delta-\text { Lorent } z \text { force (proposed) } \\
& \longrightarrow \square-\text { forced amplitude }
\end{aligned}
$$

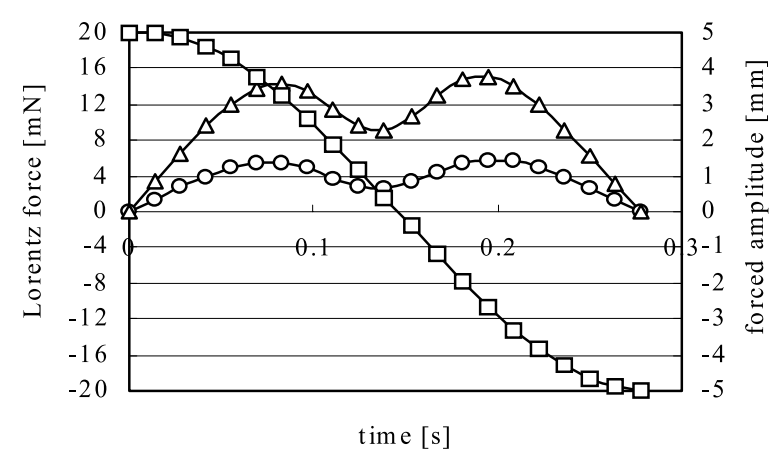

Fig. 9. Comparison of the Lorentz force between the proposed and conventional models

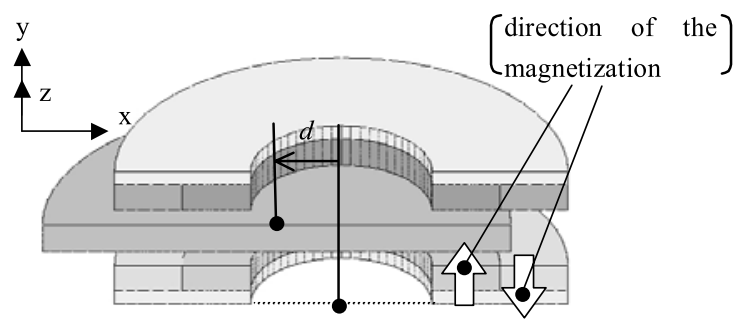

$d=4.75 \mathrm{~mm}$ (initial displacement)

Fig. 10. Basic construction of the sandwiched model

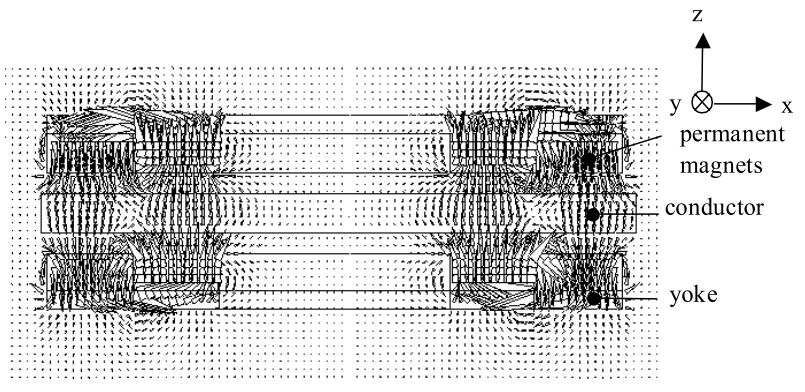

(a) flux density vectors

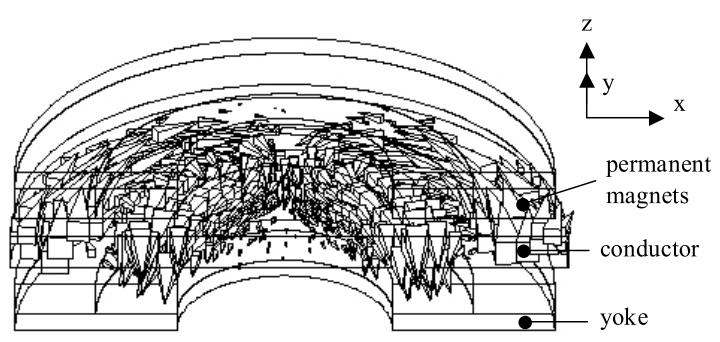

(b) eddy current density vectors

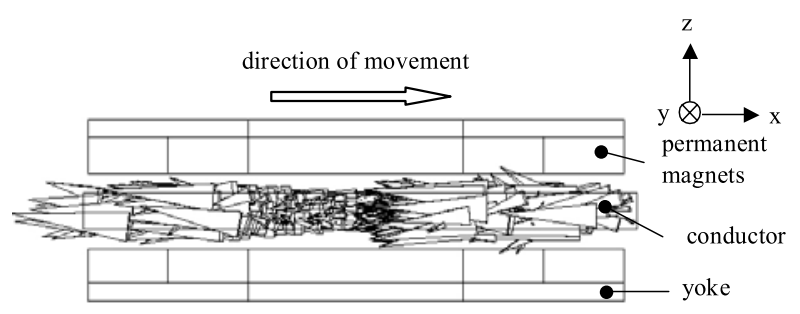

(c) Lorentz force density vectors

Fig. 11. Distributions of flux density vectors, eddy current density vectors and Lorentz force density vectors of the sandwiched model

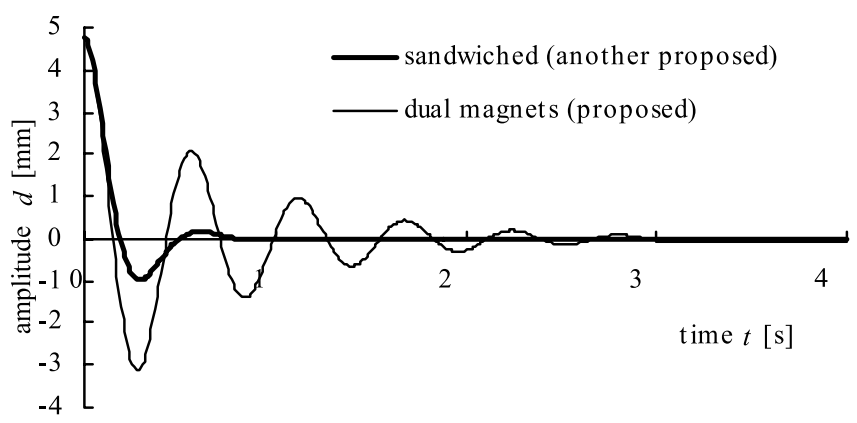

Fig. 12. Comparison of the dynamic response between two proposed models 
magnetic flux vertically flows in the conductor, and the eddy current effectively flows in the wide area of the conductor. Therefore, Lorentz force greatly increases because the force vectors mostly turn to an opposite direction of the moving conductor. Fig. 12 shows the computed dynamic response of the sandwiched model compared with that of the dual magnets model above mentioned. From this figure, it is found that the sandwiched model can be amazingly controlled (within one second).

\section{Conclusions}

In this paper, the dynamic response of the laser marker, which has damping mechanism by the eddy current force, was analyzed using the 3-D FEM. As a result, the accuracy of the analysis method was confirmed by the measurement. Furthermore, a few effective structures were presented in order to decrease the oscillation of the pendulum quickly, and the effectiveness of them was clarified.

(Manuscript received Jan. 21, 2005, revised June 22, 2005)

\section{References}

( 1 ) K. Yoshimura, K. Nakamura, T. Yasuda, and T. Honda: "Portable Laser Maker for Construction Use", Technical Report of Matsushita Electric Works, pp.22-27 (2002)

( 2 ) T. Yamaguchi, Y. Kawase, H. Shiomoto, and K. Hirata: "3-D Finite Element Analysis of Dynamic Characteristics of Twin-Type Electromagnetic Relay", IEEE Trans. Magn., Vol.38, No.2, pp.361-364 (2002)

( 3 ) Y. Kawase, T. Yamaguchi, M. Yoshida, and K. Hirata: "3-D Finite Element Analysis of Rotary Oscillatory Actuator Using A New Auto-Mesh Coupling Method", IEEE Trans. Magn., Vol.37, No.5, pp.3711-3714 (2001)

( 4 ) Y. Hasegawa, K. Hirata, T. Ota, Y. Kawase, T. Yamaguchi, and T. Eguchi: "A Study on Damping Mechanism Using Eddy Current Effect", The Papers of Joint Technical Meeting on Static Apparatus and Rotating Machinery IEE Japan, SA-04-26/RM-04-26 (2004)
Tomohiro Ota

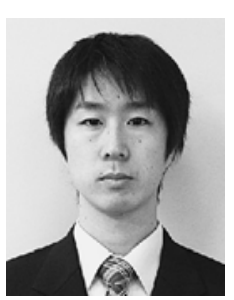

Member) was born in Gifu, Japan, on March 18, 1975. He received a B.S. degree in 1997 and M.S. degree in 1999 from Gifu University. He is presently a chief engineer at Matsushita Electrics Works, Ltd., and has worked on development of electromagnetic devices.

Tadashi Yamaguchi (Member) was born in Gifu, Japan, on July 3,

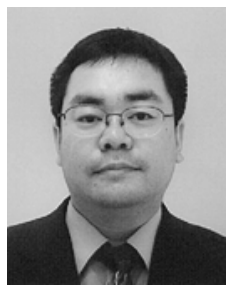
1968. He received a Dr. Eng. degree from Gifu University in 1996 . He was an assistant professor of electrical engineering at Chubu University in 1996, and became a lecture in 1998. He joined Gifu University in 1999. He is presently an associate professor in the department of information science at Gifu University from 2003. He had been engaged in development of numerical analysis of magnetic field and application. IEEE member.

Yoshihiro Kawase (Senior Member) was born in Gifu, Japan, on

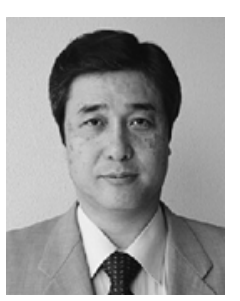
April 24, 1956. He received a M.S. degree from Okayama University in 1981, and a Dr. Eng. degree from Kyoto University in 1986. He was an assistant professor at Okayama University from 1981 to 1985. He joined Gifu University in 1985 , and became an associate professor in 1988 . He is presently a professor in the department of information science at Gifu University from 1997. He had been engaged in development of magnetic field analysis using the 3-D finite element method. IEEE member.

Takafumi Eguchi (Non-member) was born in 1982. He received a

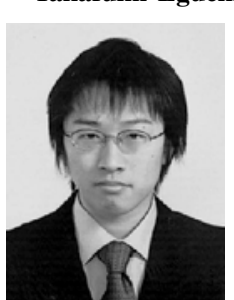
B.S. degree from Gifu University in 2004. He is presently a student of master's course at Gifu University and has studied on numerical analysis of magnetic field.

Hiroyuki Kodama (Non-member) was born in 1982. He received

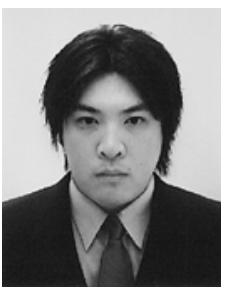
a B.S. degree from Gifu University in 2004. He is presently a student of master's course at Gifu University and has studied on numerical analysis of magnetic field.
Yuya Hasegawa (Non-member) was born in 1976. He received the

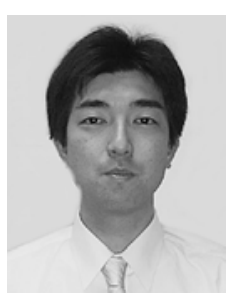

B.E. degree in 1999 and M.E. degree in 2001 from Doshisha University. He joined the R\&D lab., Matsushita Electric Works Ltd. in 2001, where he has been engaged in research and development of electromagnetic applied actuators and sensors. 